Check for updates

Cite this: RSC Adv., 2017, 7, 53822

Received 27th September 2017 Accepted 10th November 2017

DOI: 10.1039/c7ra10702a

rsc.li/rsc-advances

\section{Oxygenated graphene quantum dots (GQDs) synthesized using laser ablation for long-term real- time tracking and imaging $\dagger$}

\author{
Ashwin Kumar Narasimhan, (D) ab Swathi Lakshmi B, ${ }^{a}$ Tuhin Subhra Santra, ${ }^{a}$ \\ M. S. Ramachandra Rao ${ }^{b}$ and Ganapathy Krishnamurthi*a
}

\begin{abstract}
Fluorescence probes are essential for in vivo molecular imaging as well as cell tracking applications. Probes that emit in the far red wavelength penetrate better through biological tissue and the autofluorescence background is reduced at higher wavelengths, making such probes highly desirable. We report the application of Graphene Quantum Dots (GQDs) as efficient fluorescence probes for single cell tracking using high-resolution confocal microscopy as well as a potential in vivo fluorescent imaging agent. High-quality water soluble GQDs were synthesized by ablating highly oriented pyrolytic graphite (HOPG) in liquid using a nanosecond pulsed laser. Refluxing GQDs at $200{ }^{\circ} \mathrm{C}$ for 20 minutes and 1 hour produces excitation independent broad emission peaking at $600 \mathrm{~nm}$ and excitation dependent colortunable emission, respectively. These emission properties can be attributed to the functional groups such as carboxyl and hydroxyl groups on the surface or edges. MTT assays with the breast cancer (MCF7) cell line suggest that $20 \mathrm{~min}$ and $1 \mathrm{~h} \mathrm{GQDs}$ had $80 \%$ viability post incubation with concentrations as high as $2 \mathrm{mg} \mathrm{mL}^{-1}$. The paper also explores the molecular tracking functionality of GQDs. Fluorescence microscopy showed that MCF-7 cells incubated for up to 48 hours with GQDs, internalized the GQDs, by caveolae-mediated endocytosis without any targeting moiety. In addition, high-resolution Z stacking and 3D confocal microscopy of a single live MCF-7 cell confirm successful uptake of GQDs into the cytoplasm by endocytosis. Fluorescence imaging of GQDs loaded in polyacrylamide gel and subcutaneously implanted near the thoracic region of an euthanized mice was done to explore the feasibility of in vivo imaging using GQDs. Deep red fluorescence $(610 \mathrm{~nm}$ emission filter) was observed from the implanted region with relatively low autofluorescence background.
\end{abstract}

\section{Introduction}

Organic fluorescent molecules have been commonly used for studying biomolecular processes as well as for labeling and tracking live cells. They are prone to photobleaching and fast clearance. ${ }^{1-3}$ Semiconductor quantum dots like CdSe, HgTe, InAs and $\mathrm{HgCd}$ were seen as an efficient alternative as they do not photobleach like organic dyes and can be retained in vitro for a longer time. ${ }^{2}$ However, these were shown to be toxic to cells, making them unsuitable for long-term cell tracking and imaging studies., ${ }^{4,5}$ A viable alternative is the new class of nano carbon called graphene quantum dots. ${ }^{6}$ Since graphene is mostly carbon, it is biocompatible and has other desirable stability properties making them suitable for long-term cell imaging studies. ${ }^{7-10}$

${ }^{a}$ Department of Engineering Design, IIT Madras, Chennai, India-600036. E-mail: gankrish@iitm.ac.in

${ }^{b}$ Nanofunctional Materials Technology Centre (NFMTC), Material Science Research Centre, Department of Physics, IIT Madras, Chennai, India-600036

† Electronic supplementary information (ESI) available. See DOI: 10.1039/c7ra10702a
Recent works report different techniques used to synthesize GQDs and explore several bio-imaging applications. The focus was to synthesize GQDs appropriate for biological imaging applications. ${ }^{11}$ Typically one would require emission in the far red or infrared since autofluorescence dominates in the visible spectrum. ${ }^{12}$ The other essential requirement is that of biocompatibility over an extended period since longitudinal imaging studies are now increasingly common in both fundamental bioscience and medicine research. ${ }^{13-15} \mathrm{Jim}$ Kim et al., have synthesized GQDs for tracking and imaging of stem cells and demonstrated the efficiency of GQDs in both in vitro and in vivo for stem cell therapy applications. ${ }^{16}$ Kumawat et al. showed that one step preparation of GQDs from Mangifera indica exhibited temperature sensitive emission and could be used in nano thermometric applications. ${ }^{17}$ Jinli $\mathrm{Zhu}$ et al., developed GQDs by free radical polymerization of oxygen-containing aromatic groups using UV radiation. They have also demonstrated multicolor imaging in both in vitro and in vivo applications. ${ }^{18}$ Recently, there is increased interest in long-term live single cell imaging. In addition to challenges in designing imaging hardware and sophisticated post-processing, an 


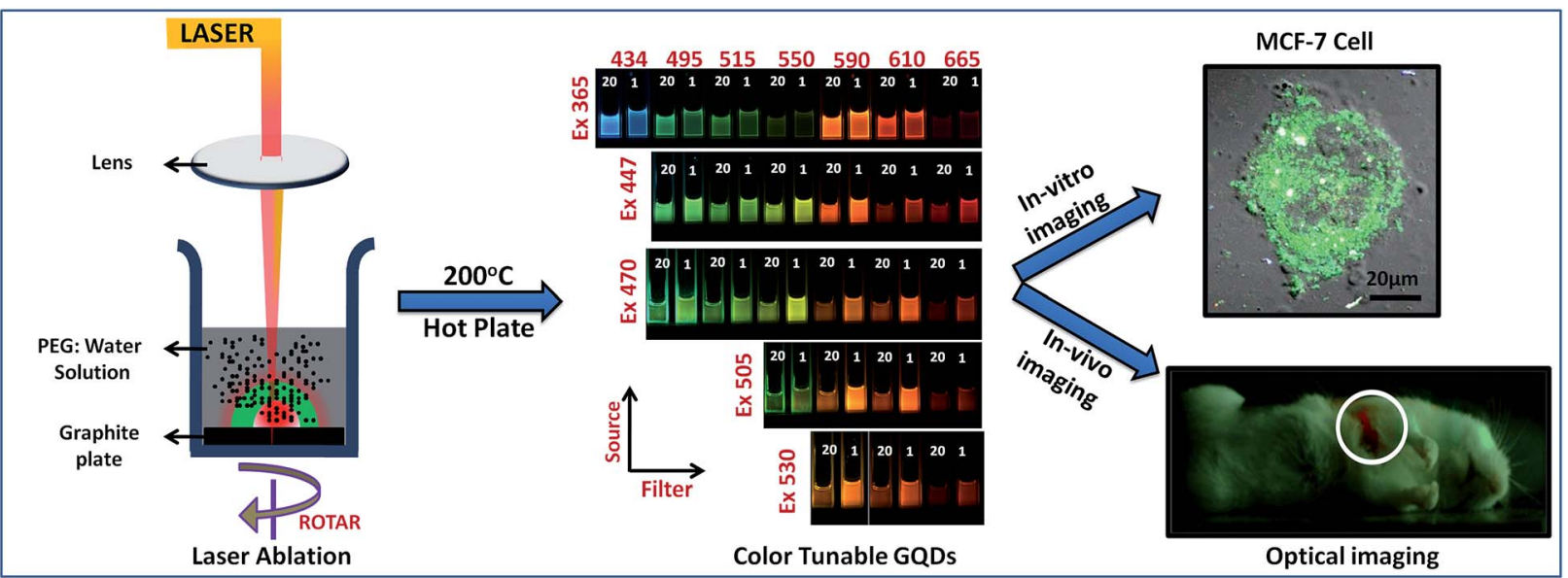

Scheme 1 Nanosecond pulse laser ablation of graphite substrate in liquids produce color tunable GQDs which can be used for tracking and imaging of MCF-7 cancer cells (a). Color tunable emission from synthesized GQDs excited at different wavelengths using commercial LED sources and captured with corresponding emission filters using a RGB CMOS camera. Two cuvettes consist of 20 min GQDs (denoted as 20 in the image) and 1 hour GQDS (denoted as 1 in the image) placed together (b). Fusion of bright light and fluorescence image of euthanized mice with gelated-GQDs implanted subcutaneously. Imaging was done in trans-illumination mode with 530 nm LED source and emitted fluorescence was captured with a long pass filter of $610 \mathrm{~nm}$ placed before the lens.

important hurdle to overcome is photo-bleaching and ensuring biocompatibility of the fluorescent probe. GQDs seem to offer an ideal solution to this problem because of their stability, resistance to photo-bleaching and ease of synthesis.

In this work we present in situ production of pegylated GQDs by laser ablation of a graphite target in PEG-water solution followed by oxygenation. The process described does not require additional reagents to obtain fluorescence GQDs. We demonstrate that GQDs synthesized in this manner have color tunable properties that are not evident in GQDs synthesized using other methods in literature. We also demonstrate novel application of these GQDs to long term single cell confocal imaging at high resolution. We demonstrate high-resolution confocal and fluorescence imaging of live cells (MCF-7) at two different time points ( 24 and 48 hours). The high-resolution fluorescence images reveal that our synthesized GQDs can enter cancer cells by caveolae-mediated endocytosis mechanism and can be retained for up to 48 hours making it a potential candidate for long-term imaging. Finally we perform small animal fluorescence imaging experiment to demonstrate an in vivo application of the synthesized GQDs with emission at $590 \mathrm{~nm}$ and $610 \mathrm{~nm}$. To the best of our knowledge, this work is unique in terms of exploring cell tracking and single cell imaging using GQDs to provide fluorescence contrast (Scheme 1).

\section{Experimental}

\section{Materials}

Synthesis of GQDs. Here we used pure highly oriented pyrolytic graphite (HOPG) plate as a source immersed in $5 \mathrm{~mL}$ of polyethylene glycol (Merck, PEG-400) and $5 \mathrm{~mL}$ Milli-Q water solution (1:2). A focused nanosecond pulsed laser (MiniliteContinuum Laser, USA) was optically directed to the graphite plate (see Fig. S1†). The laser source was operated at $1064 \mathrm{~nm}$ with specific parameters (energy $=40 \mathrm{~mJ}$, pulse duration $=6 \mathrm{~ns}$, frequency $=10 \mathrm{~Hz}) \cdot{ }^{19}$ After 30 minutes of ablation on graphite plate ( $3 \mathrm{~mm}$ thickness), single wall graphene layers and reduced GQDs were produced in the PEG-water solution. For characterization studies, the solution was centrifuged at $15000 \mathrm{rpm}$ for 30 minutes to remove large particles and graphene layers. We denote this preparation as control GQDs. Furthermore, the control-GQDs solution was refluxed at $200{ }^{\circ} \mathrm{C}$ for 20 minutes
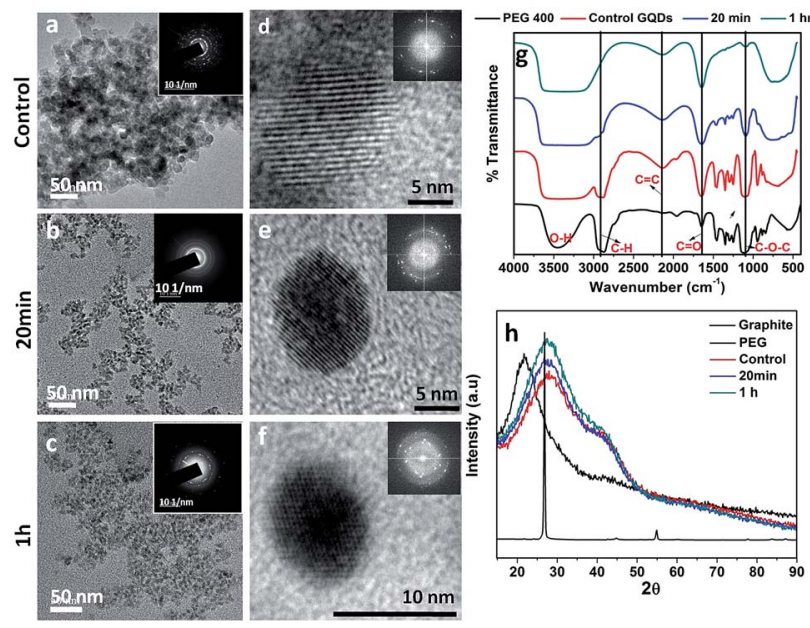

Fig. 1 TEM images of control sample GQDs (a), GQD sample refluxed for $20 \mathrm{~min}(\mathrm{~b})$ and GQD sample refluxed for $1 \mathrm{~h}(\mathrm{c})$. Inset $(\mathrm{a}-\mathrm{c})$ shows the corresponding SAED pattern. HR-TEM images of control (d), $20 \mathrm{~min}(\mathrm{e})$ and $1 \mathrm{~h}(\mathrm{f})$ samples. Inset $(\mathrm{d}-\mathrm{f}$ ) shows the SAED pattern of corresponding GQDs. The lattice patterns are clearly visible in $(d-f)$ while $(a-c)$ depicts clusters of GQDs. FT-IR spectrum of control, 20 min and $1 \mathrm{~h}$ GQDs (g). XRD spectrum of pure graphite (HOPG), polyethylene glycol (PEG-400), control, 20 min and $1 \mathrm{~h}$ GQDs particles shown in (h). 
and 1 hour. After heating for $20 \mathrm{~min}$ and $1 \mathrm{~h}$ the water evaporates completely, and the final product contains pegylated GQDs. We denote the resulting preparations as $20 \mathrm{~min}$ and $1 \mathrm{~h}$ GQDs corresponding to refluxing times of 20 minutes and 1 hour respectively.

\section{Characterization}

Morphology, chemical composition and selected area electron diffraction (SAED) of prepared samples were performed using JEOL-JEM high-resolution transmission electron microscope (HR-TEM). Atomic force microscopy (non-contact mode) was performed using park systems XEI to understand the height profile of the GQDs. Measurements were done after dispersing each sample using spin coat method on a cleaned silicon wafer. Dynamic light scattering (DLS) and zeta potential measurements were recorded using Zeta-sizer (Malvern, USA). Fourier transform infrared (FT-IR) spectrum was obtained using a Perkin-Elmer (Spectrum one) IR spectrometer. UV absorption and fluorescence spectra were recorded using Jasco-V-650 and Horiba Fluorolog-3 spectro-photometer respectively. XRD measurements were recorded using Bruker, USA (Discover-8). FT-Raman spectroscopy was performed using alpha 300, WITec, Germany (521 nm diode laser). Surface analysis was performed using X-ray photon spectroscopy (Kratos Analytical Axis 165) analyzed using monochromatic Al K-alpha source and calibrated using carbon. Fluorescence lifetime measurements were carried out to calculate decay time of GQDs using Fluorocube Jobin-Yvon excited with nanosecond lasers. Fluorescent imaging of these GQDs was performed (Nikon Eclipse Ti, Japan). Confocal imaging was performed using Carl Zeiss 810 and image processing was performed using Zen software provided by Carl Zeiss. pH stability study and quantum yield measurements were performed to study the optical properties of GQDs.

\section{Cell culture}

Human breast cancer cell lines (MCF-7, purchased from National Centre for Cell Science, Pune, India) were maintained in an incubator ( $5 \% \mathrm{CO}_{2}$ and saturated humidity) at $37{ }^{\circ} \mathrm{C}$ temperature, supplemented with Dulbecco's Modified Eagle's Medium (DMEM) containing 10\% Fetal Bovine Serum (FBS). The required amount of fresh media was replaced to maintain the healthy cells condition. When the cells grown on the culture dish reached a confluence level of $70 \%$, cells were trypsinized and recultured to maintain proper morphology and growth.

\section{Cytotoxicity assay}

Cytotoxicity study of three different GQDs was quantitatively evaluated using thiazolyl blue tetrazolium bromide colorimetric (MTT) assay. A 96-well plate with cell seeding density of $1 \times 10^{4}$ cells per well was incubated for 24 hours. Prepared control, $20 \mathrm{~min}$ and $1 \mathrm{~h}$ GQDs were incubated at various concentrations $\left(0,0.25,0.5,0.75,1,2\right.$ and $\left.5 \mathrm{mg} \mathrm{mL}^{-1}\right)$ for 24 hours. In each well, $10 \mu \mathrm{L}$ of fresh solution from the prepared $5 \mathrm{mg} \mathrm{mL}{ }^{-1}$ stock concentration of MTT was added to the cells and incubated for 4 hours. A $100 \mu \mathrm{L}$ solution of dimethyl sulphoxide (DMSO) liquid was added to each well to dissolve the MTT and placed in a microplate reader to obtain OD value (M5-Molecular Devices, USA) at 490 and $590 \mathrm{~nm}$ with DMSO as a reference. To ensure repeatability of the viability data, each sample was replicated four times to get the average value and standard deviation.

\section{Cellular imaging}

Fluorescence microscopy was used to image GQDs fluorescence emission and for cell tracking. Initially, cells were incubated for 24 and 48 hours (seeding density of $5 \times 10^{4}$ cells per well in a 24 well plate) with control, $20 \mathrm{~min}$ and $1 \mathrm{~h}$ GQDs at concentration of $2 \mathrm{mg} \mathrm{mL} \mathrm{m}^{-1}$. After incubation, cells were washed with fresh PBS and then, $4 \%$ paraformaldehyde was added to fix the cells for $10 \mathrm{~min}$ at room temperature in a shaking incubator. Further cells were washed thrice with PBS and fluorescence images were captured.

For confocal imaging, the cells were cultured in sterile coverslips (sonicated, acid washed and UV treated) and incubated overnight. After cells strongly adhered on the coverslip, it was placed on a glass slide and the ends were sealed. Highresolution confocal imaging was performed with a step size of $500 \mathrm{~nm}$ ( $\mathrm{Z}$ stacking). The acquired images were post-processed to visualize the localization of GQDs inside the live cells.

\section{In vivo imaging}

Acrylamide gel mixed with GQDs was implanted subcutaneously at a $2 \mathrm{~mm}$ depth in the thoracic region of an euthanized mouse. A small portion of the skin near the upper limbs was cut open, and the GQDs mixed with gel was placed below it. Acrylamide gel is transparent, easy to prepare, mimics muscle properties and can be made into a gel easily. Imaging was performed using our in-house developed optical imaging system. The imaging system consists of a commercial light emitting diode (LED) source (530 nm) (Luxeon, USA). ${ }^{20}$ The diverging light from the LED is optically coupled to a fiber optic cable using a plano-convex lens (Thor labs Inc., USA). The free end of the fiber optic cable was placed in contact with the skin. The emitted fluorescence was detected using a cooled CMOS RGB camera (Point Grey, Canada) coupled to a Nikkor f/1.4 lens (Nikon, Japan). Long pass emission filters with cut off wavelengths of 590 and $610 \mathrm{~nm}$ were placed in front of the lens during image acquisition. An anatomical reference image is taken without a filter and with an exposure time of 1 second using ambient light. Fluorescence imaging was performed in trans-illumination mode with an exposure time of 31 seconds and an emission filter $(690 \mathrm{~nm})$ in front of the camera lens. ${ }^{21}$

\section{Results and discussions}

High quality pegylated GQDs with optical properties suitable for biomedical imaging applications were synthesized using liquid pulse laser ablation. ${ }^{22}$ A 1064 nm LASER was able to ablate the surface of a graphite plate immersed in liquid via thermal relaxation process. ${ }^{23,24}$ Ablation of graphite plate resulted in the formation of large sheets of graphene layers (single and multiwall) and GQDs in solution (see Fig. S2 $\dagger$ ). ${ }^{23}$ The laser interaction 
at the interfacial region of graphite and liquid confinement generates high temperature $(0-4000 \mathrm{~K})$ and pressure (0-10 GPa) resulting in material vaporization and ejection of surface material. ${ }^{22,25,26}$ To separate out the GQDs, laser ablated solution was centrifuged at high speed $(15000 \mathrm{rpm})$ where the larger particles and graphene layers settled down at the bottom and supernatant was collected. The synthesized particles in PEGwater solution emitted a low-intensity green fluorescence signal when excited with $365 \mathrm{~nm}$ UV lamp confirming the presence of GQDs. ${ }^{24}$ GQDs refluxed at $200{ }^{\circ} \mathrm{C}$ for $20 \mathrm{~min}$ and $1 \mathrm{~h}$, had a high fluorescence emission due to quantum confinement effects and increased oxidation state in the edges (Fig. 1). ${ }^{27}$

Surface morphology and size of GQDs determined using high-resolution transmission electron microscopy (HR-TEM), revealed crystalline spherical particles with a size range of 2$10 \mathrm{~nm}^{28}$ FFT of synthesized GQDs showed definite lattice spacing of 0.24 and $0.33 \mathrm{~nm}$ corresponding to (100) diffraction plane of pure graphite (Fig. 1a-f). ${ }^{23}$ The change in lattice spacing implies an increase in the oxidation state between the layers and on the surface edges of GQDs. SAED patterns suggest that overall, particles were in polycrystalline structure and for a single particle hexagonal SAED pattern confirmed the presence of GQDs (inset images of Fig. 1a-f). TEM of the control GQDs showed that the ablated GQDs adhered to the entangled PEG chains. TEM images of $20 \mathrm{~min}$ and $1 \mathrm{~h}$ GQDs suggest that PEG chains were unfolded and coated with GQDs. ${ }^{29}$ In addition, atomic force microscopy (AFM) measurements indicate that the laser ablated particles possess both graphene layers and graphene dots. Lateral dimension was found to be in the range than 3-4 nm suggesting that the presence of PEG chains might increase the height of the GQDs (see Fig. S2 $\uparrow$ ). ${ }^{30}$

The size distributions of the GQDs were quantified using dynamic light scattering. Results were consistent with qualitative TEM images. The control, $20 \mathrm{~min}$ and $1 \mathrm{~h}$ GQDs had an average size of $10 \mathrm{~nm}$. The polydispersive index of GQDs had a higher value due to the presence of particles with varying size from $2-10 \mathrm{~nm}$ and was further confirmed by TEM images. Control, $20 \mathrm{~min}$, and $1 \mathrm{~h}$ GQDs had zeta potentials of $0,-9.8$ and $-8.5 \mathrm{mV}$ respectively (see Fig. S1†). Control GQDs with PEG had no surface potential but refluxed GQDs showed a higher negative zeta potential. Refluxing the GQDs changes the zeta potential to negative charge possibly due to the breakage of PEG bonds and protruding carboxyl and hydroxyl groups on the surface of GQDs.

Fourier transform infrared (FTIR) spectra (Fig. 1g) of PEG400 and GQDs were recorded to characterize the functional derivatives of GQDs. In GQDs, broad absorption band ranging from $3100-3550 \mathrm{~cm}^{-1}$ confirmed the increase in the degree of hydroxyl groups compared to PEG. ${ }^{17,31,32}$ The refluxed GQDs peak around $1648 \mathrm{~cm}^{-1}$ was higher due to the formation of carboxyl groups $(\mathrm{C}=\mathrm{O})$ on the surface/edges. The energy levels corresponding to these carboxyl groups lead to color tunable quantum dots. Similarly, peak around $1020 \mathrm{~cm}^{-1}$ (C-O-C) gradually decreased going from control to refluxed GQDs. ${ }^{29,32,33}$ FTIR spectra peak around $2900 \mathrm{~cm}^{-1}$ gradually decreased going from control to refluxed GQDs due to the breaking of the PEG chains adhered to the surface of GQDs. This breaking of $\mathrm{C}-\mathrm{H}$ bonds leads to change in zeta potential from positive to negative which is also consistent with $\mathrm{C}=\mathrm{O}$ formation, on the surface of the dots. Spectral peak at $2128 \mathrm{~cm}^{-1}(\mathrm{C}=\mathrm{C}-\mathrm{H}$ terminal bending vibration) confirmed that the ablated particles from graphite have graphene structure.

Fig. $1 \mathrm{~h}$ represents the X-ray diffraction (XRD) spectrum of pure graphite (HOPG) which shows a sharp $002\left(2 \theta=26.95^{\circ}\right)$ and $004\left(2 \theta=54.9^{\circ}\right)$ peak. Control, $20 \mathrm{~min}$, and $1 \mathrm{~h}$ GQDs show a broad diffraction peak around $26.95^{\circ}$ with a similar lattice spacing of graphite, confirming the presence of polycrystalline GQDs..$^{34}$ The characteristic peak at $100\left(2 \theta=43^{\circ}\right)$ and $110\left(2 \theta=77.8^{\circ}\right)$ of graphite implies stacked $2 \mathrm{D}$ in-plane symmetry arrangement of graphene layers. ${ }^{33}$ These peaks disappeared after laser ablation. In GQDs, broad hump in the spectrum corresponds to the turbostratic band of disordered graphene layers that would arise with increase in oxygenation and due to the presence of PEG. ${ }^{35}$ To understand the chemical structure of GQDs, FT-Raman and XPS were performed (see Fig. S3†).

Energy levels of the graphene can be divided into different absorption bands corresponding to $\mathrm{n}-\pi^{*}(\mathrm{C}=\mathrm{O})$ and $\pi-\pi^{*}$ (aromatic $\mathrm{C}-\mathrm{C}$ ). Overall electronic structure of GQDs with strong $\pi$ electron system could be altered by surface/edge oxidation. Another phenomenon contributing to emission from GQDs is the quantum size effects..$^{32,35,36}$ Absorption spectra of control GQDs had energy bands at 225 (C-C) and $285 \mathrm{~nm}(\mathrm{C}=$ O) and a small hump at $256 \mathrm{~nm}(\mathrm{C}=\mathrm{C}) \cdot{ }^{35} \mathrm{GQDs}$ refluxed for 20 minutes had $\lambda_{\max }$ at 220, 285 and a small hump around $320 \mathrm{~nm}$. For $1 \mathrm{~h}$ GQDs had $\lambda_{\max }$ at 225 , and $265 \mathrm{~nm}$ (Fig. 2a). ${ }^{36-38}$ Control GQDs had a color-tunable emission at different excitations between 350 and $590 \mathrm{~nm}$ (Fig. 2b). Due to molecular modification, $\lambda_{\max }$ in the absorption spectrum of 20 min GQDs had a red shift when compared to control GQDs (Fig. 2c). Refluxed
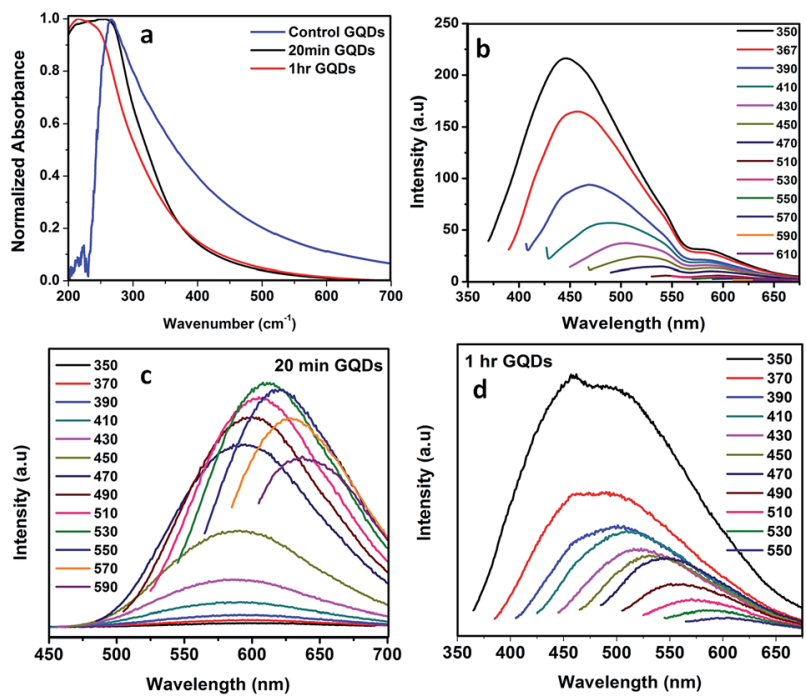

Fig. 2 Absorbance spectra of control, $20 \mathrm{~min}$ and $1 \mathrm{~h}$ GQDs (a), emission spectrum of control GQDs (b), photoluminescence spectrum of excitation independent near red emission properties 20 min GQDs (c), photoluminescence spectrum of excitation dependent $1 \mathrm{~h}$ GQDs (d). 
20 min GQDs were oxidized and had increased fluorescence intensity due to the incorporation of energy level corresponding to $\mathrm{C}=\mathrm{O}$. Emission peaks between 575-625 $\mathrm{nm}$ were observed for all excitation wavelengths with the maximum fluorescence intensity at $600 \mathrm{~nm}$ when excited at $530 \mathrm{~nm}$. The emission spectra of $1 \mathrm{~h}$ GQDs had intense peaks at 450 and $470 \mathrm{~nm}$ when excited with 350 and $370 \mathrm{~nm}$ respectively. Fluorescence intensity of GQDs gradually reduced, when excited above $400 \mathrm{~nm}$ (Fig. 2d). Compared to $20 \mathrm{~min}$ and control GQDs, $1 \mathrm{~h}$ GQDs showed excellent broad excitation independent emission ranging from 450 to $625 \mathrm{~nm}$. However, the intensity of emission peaks was diminished at higher wavelengths. This full-color tunable emission of $1 \mathrm{~h}$ GQDs could be due to the surface or edge effects and quantum confinement effects. The advantage of using nanosecond laser is that they are capable of producing single/few layered GQDs without any pores or holes. ${ }^{23}$ So, emission could be only due to introduction of carboxyl groups through refluxing of GQDs. Smaller GQDs contribute emission at lower wavelengths due to the presence of functional groups in the surface/edges of quantum dots. While the emission at longer wavelengths was influenced by the larger GQDs and some minor effects from the surface states. These functional groups introduce new energy levels results in higher possibilities of electron transition within the GQDs. ${ }^{32}$

\section{Fluorescence stability}

Fluorescence stability of $20 \mathrm{~min}$ and $1 \mathrm{~h}$ GQDs were investigated after dispersing in different $\mathrm{pH}$ conditions $(1,7.4$ and 11 , shown in Fig. $\mathrm{S} 4 \dagger$ ). At various $\mathrm{pH}$ conditions, GQDs produced independent emission features. Emission intensity of GQDs varied with $\mathrm{pH}$ was due to the deprotonation of carboxyl and hydroxyl groups of GQDs in free zig-zag sites which causes a change in Fermi level. ${ }^{39}$ Emission intensities of 20 min GQDs were high in physiological and basic condition compared to acidic

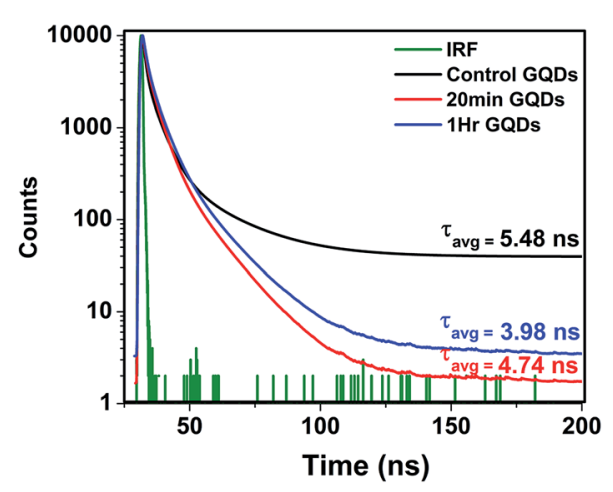

Table 1

\begin{tabular}{|c|c|c|c|c|}
\hline SAMPLE & $\tau_{1}$ & $\boldsymbol{\tau}_{\mathbf{2}}$ & $\boldsymbol{\tau}_{\text {avg }}$ & QY \\
\hline Control GQDs & $1.68 \mathrm{~ns}(54.1 \%)$ & $10 \mathrm{~ns}(45.8 \%)$ & $5.48 \mathrm{~ns}$ & $24.90 \%$ \\
\hline 20min-GQDs & $2.19 \mathrm{~ns}(59.4 \%)$ & $8.7 \mathrm{~ns}(40.5 \%)$ & $4.74 \mathrm{~ns}$ & $47.16 \%$ \\
\hline 1Hr-GQDs & $2.3 \mathrm{~ns}(56.4 \%)$ & $9.5 \mathrm{~ns}(43.6 \%)$ & $3.98 \mathrm{~ns}$ & $12.80 \%$ \\
\hline
\end{tabular}

Fig. 3 Fluorescence life time of control, 20 min, 1 hour GQDs, and IRF. Life time increases with increasing reflux time indicating presence of new carboxyl groups at surface or edges (a). Table 1 represents the lifetime and quantum yield measurements (bottom). condition. In case of $1 \mathrm{~h}$ GQDs, at physiological condition the emission intensity was lower when compared to the acidic and basic condition. In cancer cells, $\mathrm{pH}$ values vary from 5.5 to 7 , where $20 \mathrm{~min}$ GQDs seems to have higher emission intensity and can potentially be used for in vivo imaging applications. ${ }^{40}$

\section{Quantum yield}

Table 1 represents the estimated quantum yields (QY) for control, $20 \mathrm{~min}$ and $1 \mathrm{~h}$ GQDs dispersed in PBS (using ATTO-390, ATTORho $6 \mathrm{G}$, and ATTO-390 as references with excitation wavelengths of 535 and $390 \mathrm{~nm}$, respectively). GQDs-20 min had a higher quantum yield when compared with control GQDs. Similarly, when refluxing time was increased from $20 \mathrm{~min}$ to $1 \mathrm{~h}$, we observe a gradual reduction in quantum yield (a difference of $12.8 \%$ ). The quantum yields were calculated using an open software named Fluortools. ${ }^{41}$ At $\mathrm{pH} 7.4,1 \mathrm{~h}$ GQDs had a decrease in quantum yield compared to 20 min GQDs possibly due to the $\mathrm{pH}$ explained in the previous section.

\section{Lifetime measurements}

Lifetime decay. Lifetime decay curves of luminescent quantum dots of instrument response function (IRF), control, $20 \mathrm{~min}$ and $1 \mathrm{~h}$ GQDs are shown in Fig. 3. IRF was collected using a scatterer excited at $370 \mathrm{~nm}$ using LED laser light source and the full width half maximum (FWHM) of the detector response was $\sim 1$ ns. Decay measurements were carried out at room temperature by exciting GQDs at $370 \mathrm{~nm}$ (laser diode) and the emission recorded around $500 \mathrm{~nm}$ by time-correlated single photon technique. Furthermore, obtained results were fit to a double exponential function to determine the lifetime measurements. Control GQDs had an exponential decay time of $1.68 \mathrm{~ns}$ (54\%) and $10 \mathrm{~ns}(45 \%)$ with an average lifetime of $5.48 \mathrm{~ns}$. The control decay curve goes down to about 100 counts while the other two curves ( $20 \mathrm{~min}$ and $1 \mathrm{~h}$ GQDs) go down to about 10 counts. Both of these counts (10 and 100) are statistically insignificant compared to the peak values ( $\sim 000$ counts) of the individual decay curves. The $20 \mathrm{~min}$ GQDs had a lifetime of 2.19 (59\%) and $8.7 \mathrm{~ns}(40 \%)$ with an average lifetime of $4.74 \mathrm{~ns}$. Similarly, for the $1 \mathrm{~h}$ GQDs, the decay rates were 2.3 (56\%) and $9.5 \mathrm{~ns}$ (43\%) with an average lifetime of $3.98 \mathrm{~ns}$. Compared to the control, $20 \mathrm{~min}$ and $1 \mathrm{~h}$ GQDs the fluorescence lifetime gradually decreases due to the introduction of new carboxyl groups at surface/edges of GQDs as explained in the previous section. Fitted lifetime measurements are summarized in the Table 1 . The lifetime of these quantum dots in nanosecond suggests that the synthesized GQDs are appropriate for bio-imaging applications. ${ }^{\mathbf{4 2 - 4 4}}$

\section{Cellular imaging}

Fluorescence images of MCF-7 cells (incubated with GQDs) are shown in Fig. S5. $\uparrow$ The images clearly indicated blue and green color emission produced from GQDs with corresponding excitation wavelengths of 365 and $405 \mathrm{~nm}$. The images provide visual confirmation that GQDs binds with the cellular membrane, and there might have been some uptake over the incubation period without any targeting moiety. Moreover, 
these results validate the GQDs fluorescence emission properties that were observed in the solution.

Confocal imaging results confirmed the intracellular uptake of the GQD into cells. The cells were incubated with $2 \mathrm{mg} \mathrm{mL}^{-1}$ of GQDs for 24 and 48 hours, and the acquired images are shown in Fig. 4. To understand cellular uptake, we performed confocal scanning at a single cell level after 24 hours incubation (Fig. 5). The results confirmed the presence of GQDs in the cytoplasm but not in the nucleus. At various depths, the GQDs were distributed near the cells walls and sometimes near the interior of the cells. Similarly, 3D imaging of single cell confirms the complete cellular uptake of GQDs without any targeting agent. Thus, the confocal microscopy images of cells incubated with $2 \mathrm{mg} \mathrm{mL}^{-1}$ provided evidence of cellular uptake as well high-intensity fluorescence emission from GQDs even after 48 hours (see Fig. S6 $\dagger$ ).

\section{Cytotoxicity study}

MCF-7 cells treated with control, 20 min and $1 \mathrm{~h}$ GQDs were tested using MTT assay of different concentrations $(0.25-5 \mathrm{mg}$ $\mathrm{mL}^{-1}$ ). Fig. 6 shows the percentage of cell viability at different GQDs concentrations for control, $20 \mathrm{~min}$, and $1 \mathrm{~h}$. There was significant reduction in the viability of MCF-7 cells upon control GQDs exposure and it had less of an effect in $20 \mathrm{~min}$. At $2 \mathrm{mg}$ $\mathrm{mL}^{-1}$ concentration, $20 \mathrm{~min}$ and $1 \mathrm{~h}$ GQDs, there was no significant cell death incubated for 24 hours. But for the control GQDs, there was $60 \%$ cell death even at a low concentration of $0.25 \mathrm{mg} \mathrm{mL}^{-1}$. Increase in the toxicity of MCF-7 cells, when treated with control GQDs could be due to the presence of nonoxidized graphene quantum dots that induce ROS which could lead to stress on the cells..$^{45}$ The viability of MCF-7 cells in $1 \mathrm{~h}$ GQDs was higher compared to 20 min GQDs could be because of oxidation. The ultra-small sized particles, the presence of PEG,

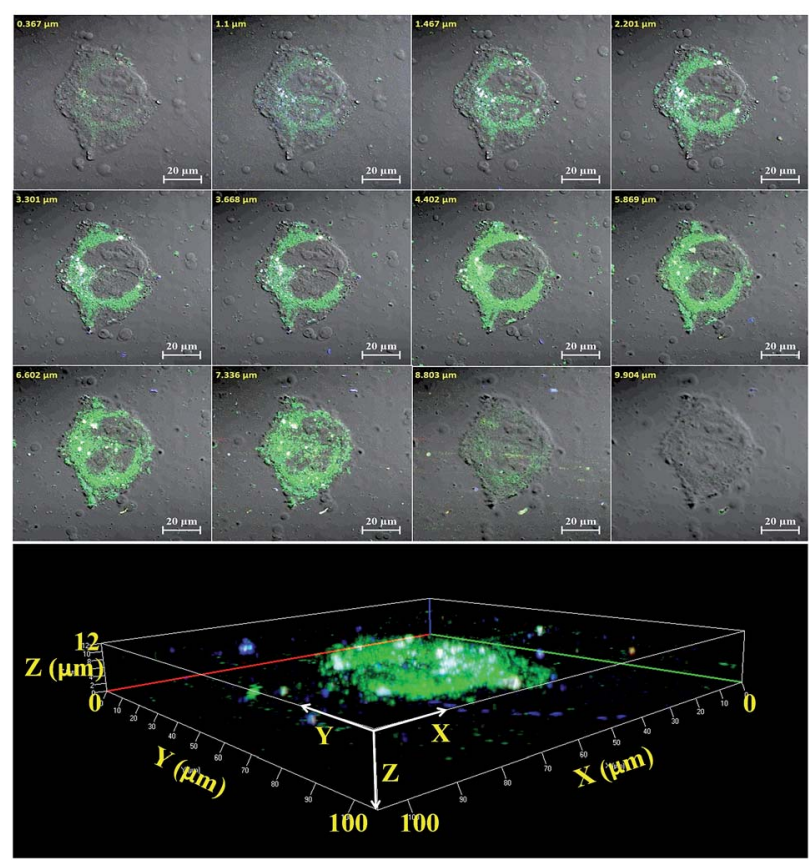

Fig. 5 High resolution single cell imaging and corresponding z-stack single cell images demonstrating localization of GQDs in cytoplasm and not in the nucleus. Scale bar: $20 \mu \mathrm{m}$. The bottom most images is the topographic stacked section of single cells.

and higher oxygen level at surface or edges can also enhance the cell viability. ${ }^{15}$ The above results indicate that GQDs are largely biocompatible and consistent with the previous reports..$^{13,46,47}$

\section{In vivo imaging}

Fig. 7a represents the ambient light image for anatomical reference while Fig. $7 \mathrm{~b}$ shows the fluorescence image. The

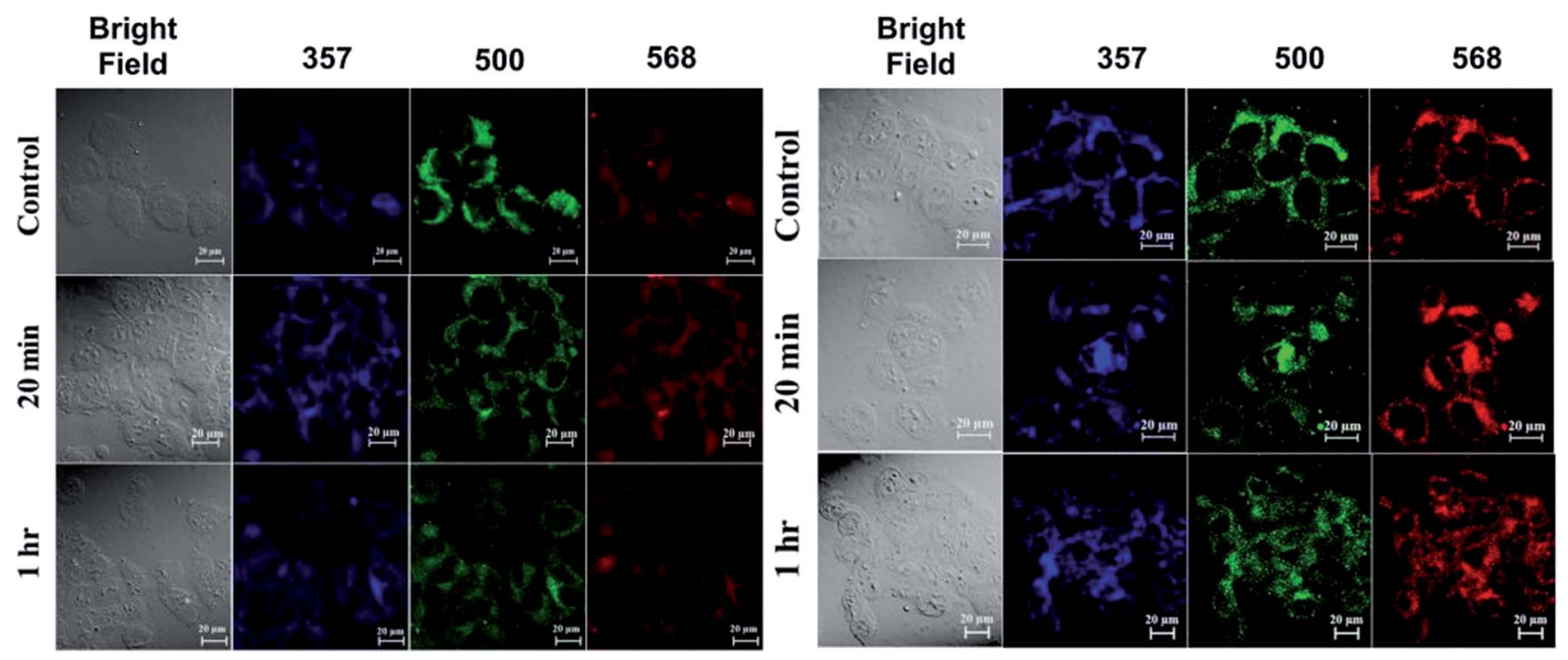

Fig. 4 Confocal images of clusters of MCF-7 cells incubated with control, 20 min and $1 \mathrm{~h}$ GQDs excited at 357, 500 and $568 \mathrm{~nm}$ and corresponding bright field images with two different incubation times of 24 (left) and 48 hours (right) respectively. Scale bar: $20 \mu \mathrm{m}$. Fluorescence images show uptake of GQDs near the cell membrane as well as in the cytoplasm. Fluorescence intensity at different wavelengths is consistent with photoluminescence measurements with strong excitation dependent fluorescence. 


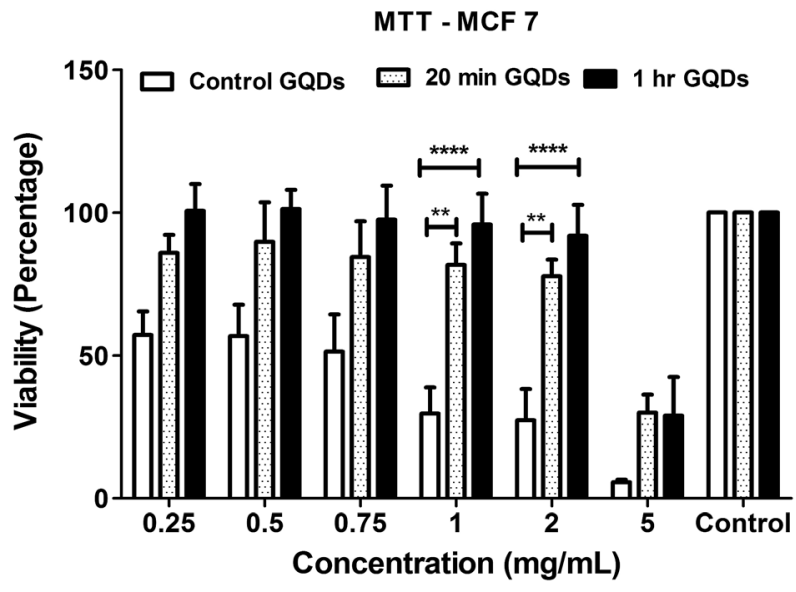

Fig. 6 Cell viability of control, 20 min and $1 \mathrm{~h}$ GQDs incubated with MCF- 7 cells (breast cancer cell line) for 24 hours. Increasing reflux time leads to increase in cell viability across all concentrations compared to control GQDs.

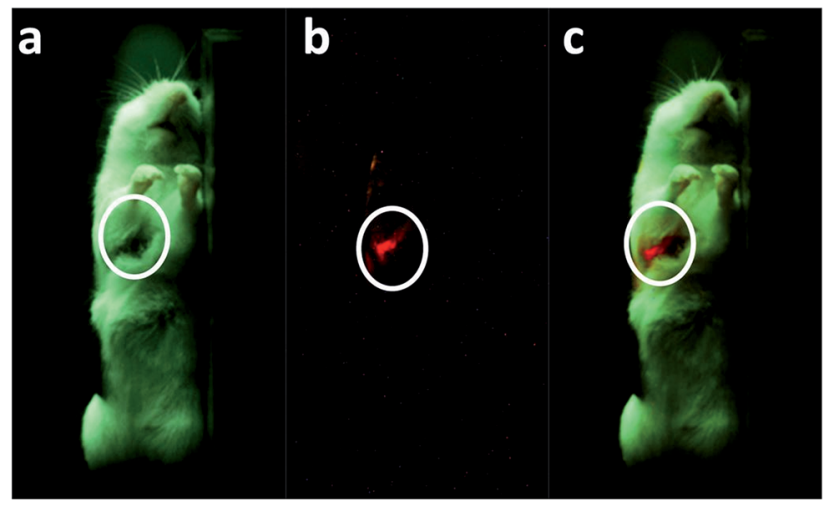

Fig. 7 In vivo imaging of GQD loaded in acrylamide gel and excited with $530 \mathrm{~nm}$ and $610 \mathrm{~nm}$ long pass emission filter. White light image of mouse with circle indicating implant region. Fluorescence image acquired with long pass filter. Blended image confirms the source of fluorescence from implanted region.

image is shown in Fig. 7c is the merged image of Fig. 7a and b. Fig. 7c shows bright red fluorescence from the GQDs located in the subcutaneous site differentiating it from the autofluorescence background. ${ }^{21}$ In this imaging study, the autofluorescence is lower compared to the fluorescence signal from GQDs because of imaging in the trans-illumination mode and due to the GQDs being placed at a shallow depth. Qualitatively, the strength of the fluorescence signal compared to the autofluorescence demonstrates that the synthesized GQDs can be used for in vivo imaging applications. Similarly, GQDs were excited with $530 \mathrm{~nm}$ and acquired using $590 \mathrm{~nm}$ emission filter (see Fig. S7†). ${ }^{48}$

\section{Conclusions}

In summary, high-quality GQDs were produced by ablating graphite plate in liquids using nanosecond pulsed laser ablation. These GQDs had properties desirable for in vivo imaging applications. Modifying the oxidation level of these quantum dots improved the optical properties by changing the quantum confinement effects. Upon refluxing the ablated particles, excitation independent emission in the near red region and excitation dependent color-tunable emission were observed. Experiments indicate that $20 \mathrm{~min}$ GQDs had strong fluorescence intensity at a $\mathrm{pH} 1-7$, suggesting that these particles might be used as fluorescent biomarkers for drug delivery application. Low cytotoxicity of GQDs over 24 hours period are also favorable for bio-imaging applications. We observed that GQDs less than $10 \mathrm{~nm}$ in size could easily enter into the cells through caveolae-mediated endocytosis process, and fluorescence intensity could be retained in the cytoplasm for over 48 hours. Confocal imaging of MCF-7 cells with GQDs not only provided evidence of cellular uptake but also demonstrated that the cell morphology remains intact even after 48 hours post incubation. Trans-illumination of gelated GQDs implanted subcutaneously in an euthanized mouse showed localization of GQDs in tissue. Overall, synthesized particles with these properties can serve as an optical contrast agent in both cell imaging and pre-clinical imaging applications.

\section{Conflicts of interest}

There are no conflicts to declare.

\section{Acknowledgements}

This work acknowledges the support of Nano Functional Materials Technology Centre (NFMTC), Material Science Research Centre (MSRC), and Department of Physics. Thanks to the interdisciplinary program, IIT-M. Thanks to Sophisticated Analytical Instruments Facilities of (SAIF) IIT-M. Thanks to Prof. Suresh Rayala, Department of biotechnology, IIT-M for performing cellular experiments and characterization of particles. We thank National Cancer Tissue Bank (NCTB), Department of Biotechnology, IITM for providing confocal scanning microscopy facility.

\section{Notes and references}

1 M. A. Walling, J. A. Novak and J. R. Shepard, Int. J. Mol. Sci., 2009, 10, 441-491.

2 U. Resch-Genger, M. Grabolle, S. Cavaliere-Jaricot, R. Nitschke and T. Nann, Nat. Methods, 2008, 5, 763-775.

3 X. Michalet, F. F. Pinaud, L. A. Bentolila, J. M. Tsay, S. Doose, J. J. Li, G. Sundaresan, A. M. Wu, S. S. Gambhir and S. Weiss, Science, 2005, 307, 538-544.

4 L. W. Zhang, W. Baumer and N. A. Monteiro-Riviere, Nanomedicine, 2011, 6, 777-791.

5 M. Bottrill and M. Green, Chem. Commun., 2011, 47, 70397050 .

6 J. Li and J. J. Zhu, Analyst, 2013, 138, 2506-2515.

7 J. Lin, X. Chen and P. Huang, Adv. Drug Delivery Rev., 2016, 105, 242-254. 
8 H. He, H. Wang, K. Li, J. Zhu, J. Liu, X. Meng, X. Shen, X. Zeng and W. Cai, Langmuir, 2016, 32, 1667-1673.

9 M. Bacon, S. J. Bradley and T. Nann, Part. Part. Syst. Charact., 2014, 31, 415-428.

10 K. V. Krishna, C. Menard-Moyon, S. Verma and A. Bianco, Nanomedicine, 2013, 8, 1669-1688.

11 Y. Du and S. Guo, Nanoscale, 2016, 8, 2532-2543.

12 J. Shi, L. Wang, J. Zhang, R. Ma, J. Gao, Y. Liu, C. Zhang and Z. Zhang, Biomaterials, 2014, 35, 5847-5861.

13 X. Yuan, Z. Liu, Z. Guo, Y. Ji, M. Jin and X. Wang, Nanoscale Res. Lett., 2014, 9, 108.

14 I. V. Pavlidis, M. Patila, U. T. Bornscheuer, D. Gournis and H. Stamatis, Trends Biotechnol., 2014, 32, 312-320.

15 Y. Chong, Y. Ma, H. Shen, X. Tu, X. Zhou, J. Xu, J. Dai, S. Fan and Z. Zhang, Biomaterials, 2014, 35, 5041-5048.

16 J. Kim, S. H. Song, Y. Jin, H. J. Park, H. Yoon, S. Jeon and S. W. Cho, Nanoscale, 2016, 8, 8512-8519.

17 M. K. Kumawat, M. Thakur, R. B. Gurung and R. Srivastava, ACS Sustainable Chem. Eng., 2017, 5, 1382-1391.

18 J. Xu, X. Shen, L. Jia, Z. Xu, T. Zhou, X. Li, T. Ma, H. Li, Y. Wang and T. Zhu, Mater. Lett., 2017, 203, 37-41.

19 S.-L. Hu, K.-Y. Niu, J. Sun, J. Yang, N.-Q. Zhao and X.-W. Du, J. Mater. Chem., 2009, 19, 484.

20 K. R. Kells, K. Y. Kong, W. B. White, C. Kaddi and M. D. Wang, 2013 IEEE Point-of-Care Healthcare Technologies (Pht), 2013, pp. 9-12.

21 M. Kleshnin, M. Shirmanova, I. Fiks, A. Orlova, V. Plekhanov, E. Zagainova, S. Lukyanov and I. Turchin, Photonics Lasers Med., 2015, 4, 85-92.

22 D. Reyes, M. Camacho, M. Camacho, M. Mayorga, D. Weathers, G. Salamo, Z. Wang and A. Neogi, Nanoscale Res. Lett., 2016, 11, 424.

23 T. N. Lin, K. H. Chih, C. T. Yuan, J. L. Shen, C. A. Lin and W. R. Liu, Nanoscale, 2015, 7, 2708-2715.

24 P. Russo, A. Hu, G. Compagnini, W. W. Duley and N. Y. Zhou, Nanoscale, 2014, 6, 2381-2389.

25 J. P. Sylvestre, S. Poulin, A. V. Kabashin, E. Sacher, M. Meunier and J. H. T. Luong, J. Phys. Chem. B, 2004, 108, 16864-16869.

26 X. D. Ren, R. Liu, L. M. Zheng, Y. P. Ren, Z. Z. Hu and H. He, Appl. Phys. Lett., 2015, 107, 141907.

27 C. Wang, L. Feng, H. Yang, G. Xin, W. Li, J. Zheng, W. Tian and X. Li, Phys. Chem. Chem. Phys., 2012, 14, 13233-13238.

28 F. Zhang, F. Liu, C. Wang, X. Xin, J. Liu, S. Guo and J. Zhang, ACS Appl. Mater. Interfaces, 2016, 8, 2104-2110.

29 S. Zhang, P. Xiong, X. Yang and X. Wang, Nanoscale, 2011, 3, 2169-2174.
30 S. Mittal, V. Kumar, N. Dhiman, L. K. Chauhan, R. Pasricha and A. K. Pandey, Sci. Rep., 2016, 6, 39548.

31 S. Zhu, J. Zhang, S. Tang, C. Qiao, L. Wang, H. Wang, X. Liu, B. Li, Y. Li, W. Yu, X. Wang, H. Sun and B. Yang, Adv. Funct. Mater., 2012, 22, 4732-4740.

32 H. Nie, M. Li, Q. Li, S. Liang, Y. Tan, L. Sheng, W. Shi and S. X. A. Zhang, Chem. Mater., 2014, 26, 3104-3112.

33 A. Bagri, C. Mattevi, M. Acik, Y. J. Chabal, M. Chhowalla and V. B. Shenoy, Nat. Chem., 2010, 2, 581-587.

34 C. Zhu, T. Y.-J. Han, E. B. Duoss, A. M. Golobic, J. D. Kuntz, C. M. Spadaccini and M. A. Worsley, Nat. Commun., 2015, 6, 6962.

35 B. Y. Chang, N. M. Huang, M. N. An'amt, A. R. Marlinda, Y. Norazriena, M. R. Muhamad, I. Harrison, H. N. Lim and C. H. Chia, Int. J. Nanomed., 2012, 7, 3379-3387.

36 T. F. Emiru and D. W. Ayele, Egyptian Journal of Basic and Applied Sciences, 2017, 4, 74-79.

37 G. S. Kumar, R. Roy, D. Sen, U. K. Ghorai, R. Thapa, N. Mazumder, S. Saha and K. K. Chattopadhyay, Nanoscale, 2014, 6, 3384-3391.

38 D. Qu, M. Zheng, P. Du, Y. Zhou, L. Zhang, D. Li, H. Tan, Z. Zhao, Z. Xie and Z. Sun, Nanoscale, 2013, 5, 12272-12277.

39 R. Ye, C. Xiang, J. Lin, Z. Peng, K. Huang, Z. Yan, N. P. Cook, E. L. Samuel, C. C. Hwang, G. Ruan, G. Ceriotti, A. R. Raji, A. A. Marti and J. M. Tour, Nat. Commun., 2013, 4, 2943.

40 S.-H. Song, M. Jang, H. Yoon, Y.-H. Cho, S. Jeon and B.-H. Kim, RSC Adv., 2016, 6, 97990-97994.

$41 \mathrm{~F}$. a|e - UV-Vis-IR Spectral Software 1.2, http:// www.fluortools.com.

42 K. Hoffmann, T. Behnke, D. Drescher, J. Kneipp and U. Resch-Genger, ACS Nano, 2013, 7, 6674-6684.

43 X. Deng, J. Sun, S. Yang, H. Shen, W. Zhou, J. Lu, G. Ding and Z. Wang, Appl. Phys. Lett., 2015, 107, 241905.

44 K. Okabe, N. Inada, C. Gota, Y. Harada, T. Funatsu and S. Uchiyama, Nat. Commun., 2012, 3, 705.

45 M. C. Mendonca, E. S. Soares, M. B. de Jesus, H. J. Ceragioli, A. G. Batista, A. Nyul-Toth, J. Molnar, I. Wilhelm, M. R. Marostica Jr, I. Krizbai and M. A. da Cruz-Hofling, Mol. Pharmaceutics, 2016, 13, 3913-3924.

46 J. Sun, Y. Deng, J. Li, G. Wang, P. He, S. Tian, X. Bu, Z. Di, S. Yang, G. Ding and X. Xie, ACS Appl. Mater. Interfaces, 2016, 8, 10226-10233.

47 L. Zhou, J. Geng and B. Liu, Part. Part. Syst. Charact., 2013, 30, 1086-1092.

48 V. Ntziachristos, J. Ripoll, L. V. Wang and R. Weissleder, Nat. Biotechnol., 2005, 23, 313-320. 